\title{
Desempeño en métodos de navegación autónoma para robots móviles
}

\author{
Alvarez Gabriela ${ }^{1}$ y Flor Omar ${ }^{2}$. \\ $\left\{\right.$ gabriela.alvarez.velasco ${ }^{1}$, omar.flor $\left.^{2}\right\} @$ udla.edu.ec \\ https://orcid.org/0000-0002-0882-3875 \\ Universidad de las Américas, Facultad de Ingeniería y Ciencias Aplicadas ${ }^{1}{ }^{2}$ \\ Quito-Ecuador
}

Recibido (03/07/20), Aceptado (21/03/20)

\begin{abstract}
Resumen: En este trabajo se presenta una comparación de los tiempos de respuesta, optimización de la ruta y complejidad del grafo en métodos de planificación de trayectoria para robots móviles autónomos. Se contrastan los desarrollos de Voronoi, Campos potenciales, Roadmap probabilístico y Descomposición en celdas para la navegación en un mismo entorno y validándolos para un número variable de obstáculos. Las evaluaciones demuestran que el método de generación de trayectoria por Campos Potenciales, mejora la navegación respecto de la menor ruta obtenida, el método Rapidly Random Tree genera los grafos de menor complejidad y el método Descomposición en celdas, se desempeña con menor tiempo de respuesta y menor coste computacional.
\end{abstract}

Palabras Clave: Optimización; trayectoria; métodos de planificación; robots móviles.

\section{Performance in autonomous navigation methods for mobile robots}

Abstract: This paper presents a comparison of response times, route optimization, and graph complexity in path planning methods for autonomous mobile robots. The developments of Voronoi, Potential Fields, Probabilistic Roadmap and Decomposition in cells for navigation in the same environment are contrasted and validated for a variable number of obstacles. Evaluations show that the path generation method by Potential Fields improves navigation with respect to the shortest route obtained, the Rapidly Random Tree method generates graphs of less complexity and the Decomposition in cells method, performs with less response time and lower computational cost.

Keywords: Optimization; trajectory; planning methods; mobile robots.

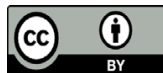




\section{I.INTRODUCCIÓN}

A pesar de que inicialmente la aplicación de los robots móviles se limitaba a las industrias manufactureras, hoy en día ocupan varios campos y cubren un amplio espectro de aplicaciones, que incluyen minería, fuerzas armadas, rescate, espacio, agricultura y entretenimiento [1]. Entre las características que distingue a un robot móvil de otro, se encuentra su capacidad de realizar una navegación exitosa, entendiéndose como tal, una adecuada, rápida y optima planificación de la trayectoria, cuya operatividad brinda un valor a la efectividad en navegación [2]. Es por esto que la selección adecuada del método de planificación de la ruta tiene especial relevancia para que el robot sea efectivo en el cumplimiento de sus objetivos [3]. En ese sentido varios investigadores han desarrollado muchas técnicas en el campo de la navegación de robots móviles y es el tema más investigado en la actualidad [4], [5]. Hay dos técnicas sugeridas que cubren todos los enfoques en la planificación de rutas de robots: la primera es la planificación de rutas global o planificación off-line y, la segunda corresponde a la planificación de rutas locales o planificación on-line [6].

La planificación de ruta global considera una trayectoria basada en un ambiente previamente establecido. Este método tiene la finalidad de producir una ruta optimizada, pero no es útil en el caso de que el escenario cuente con obstáculos desconocidos o dinámicos [7]. Al contrario, el método de planificación de ruta local no necesita en primera instancia de información del entorno, sino que va generando y recogiendo la información de los sensores integrados. En este artículo se comparan cuatro métodos de la planificación global de rutas, los cuales son: Método de Voronoi, Campos potenciales, Roadmap probabilístico y Descomposición en celdas. Estos métodos se han considerado en diversos estudios que les han brindado relevancia en el campo de la roótica.

\section{A.Método de Voronoi}

El método de Voronoi es ampliamente utilizado en la planificación multi-robot, vigilancia y cobertura de áreas. [8] El algoritmo mantiene un bajo consumo computacional [9] y se lo puede considerar uno de los mejores métodos de planeación de trayectorias debido a la seguridad que presenta la trayectoria con respecto a otros métodos existentes. Permite encontrar la estructura geométrica entre puntos ordenados o dispersos de forma aleatoria en un plano bidimensional, fue el ruso matemático Gueorgui Feodósievich Voronói quien formalizó su estudio en el año 1908. Este método se relaciona de manera directa con diversas estructuras que se encuentran en la naturaleza, como en los patrones de hojas, cortezas de árboles, grietas en el suelo causadas por erosión, cáscaras de piña e incluso en pieles de animales como la jirafa [10]. Para comprenderlo de mejor manera, se presenta la Fig. 1.

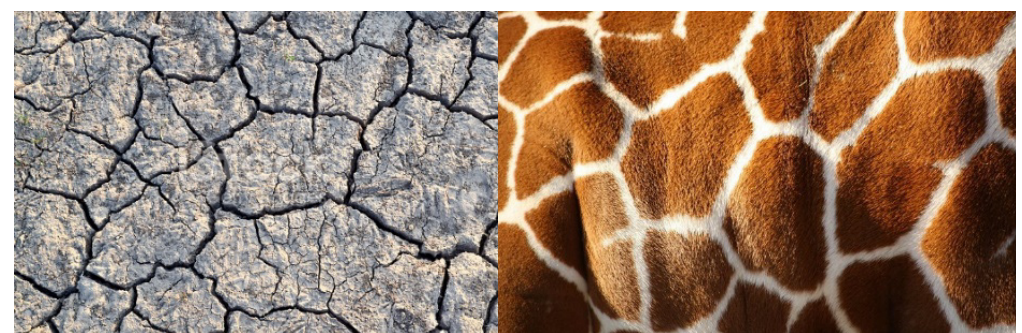

(a)

(b)

Fig. 1. Patrones de la naturaleza relacionados con los diagramas de Voronoi, (a) Grietas en el suelo, (b) Piel de jirafa.

Los diagramas de Voronoi se definen como la descomposición de un espacio métrico en regiones donde existen obstáculos, de manera que cada uno ocupa una región de este espacio, y se representan como puntos dispersos en función de su ubicación, como se observa en la Fig. 2. Los diagramas de Voronoi tienen múltiples aplicaciones en los campos de la antropología, arqueología, astronomía, biología, cartografía, geografía, matemáticas, marketing, fisiología y muchas más ciencias. 


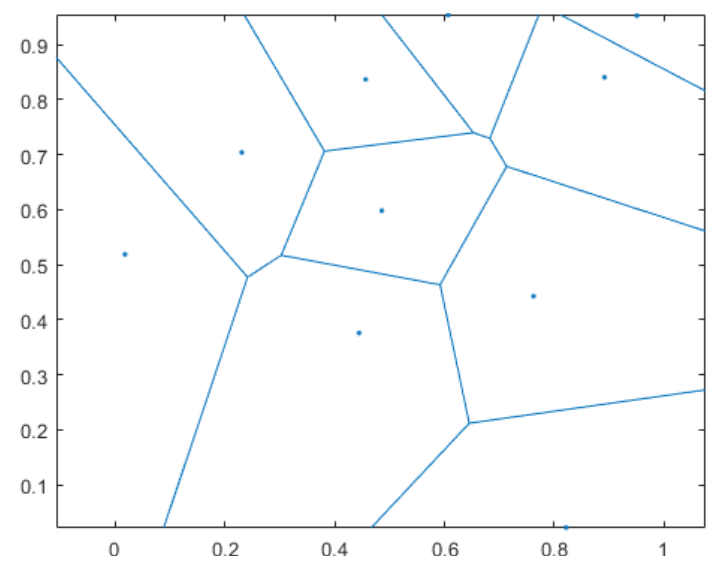

Fig. 2. Representación de diagrama de Vornoi.

En el área de la robótica el concepto fundamental de la construcción de los diagramas de Voronoi es ampliar la distancia existente entre el robot y los obstáculos al máximo. De esta manera, la red resultante es un espacio geométrico de las configuraciones que se encuentran equidistantes en los obstáculos más próximos del entorno [10]. Si se tiene un espacio físico con obstáculos determinados en las que el robot debe moverse, la manera de evitar las colisiones con este método es diseñar una trayectoria que siga el robot sobre las líneas generadas en la red de Voronoi, lo que lograría que el dispositivo se mueva siempre en medio entre dos obstáculos de la zona [11].

Para representar un área de manera más realista se posicionan los obstáculos en el espacio como polígonos en lugar de puntos [9]. En estos casos los diagramas resultantes estarán conformados por segmentos de líneas rectas y de parábolas que los rodearán como se muestra en la Fig. 3. Además, en este gráfico, se pude observar lo que se mencionó en el párrafo anterior, ya que se tienen los obstáculos poligonales y el diagrama de Voronoi en color verde, pero se tiene una trayectoria específica que enlaza el punto de inicio (verde) con el punto de llegada (rojo) en color magenta, que es la misma que recorrería el robot.

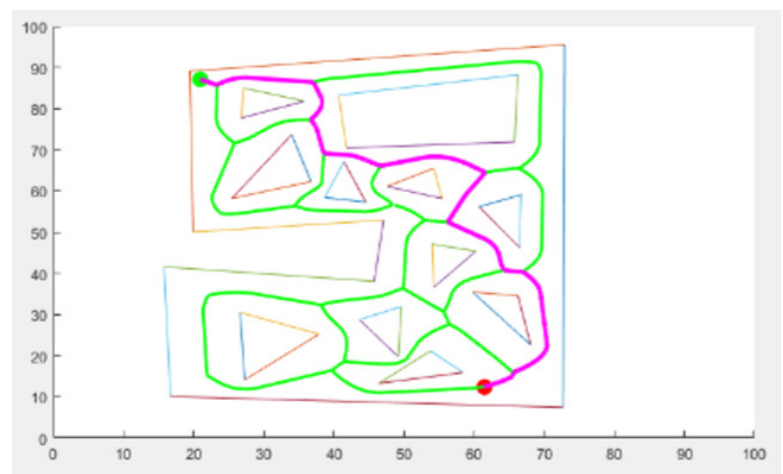

Fig. 3. Ejemplo de trayectoria óptima en un diagrama de Voronoi.

Para profundizar más en el tema se pude revisar el artículo Planeación y seguimiento de trayectorias para un robot móvil, en el cual se describen las ecuaciones y parámetros que intervienen para la realización de estos diagramas [10].

\section{B.Campos Potenciales}

El Campo Potencial se caracteriza por ser un método que expresa la matemática de generación de trayectorias de forma simple, ya que es de fácil uso y muy eficiente [12]. Su tiempo de cálculo es bajo, por lo que este algoritmo funciona en tiempo real y tiene la capacidad de ser utilizado dinámicamente debido al sencillo tratamiento de obstáculos fijos y móviles [13]. El estado del robot y la dinámica artificial puede producir una velocidad virtual y aceleración que se utilizan como una referencia instantánea para controlar la pose del robot [14].

Este método se basa en una analogía realizada entre el desplazamiento de los robots móviles y los campos poten- 
ciales eléctricos, partiendo de la premisa que el robot se desplaza como una partícula con carga eléctrica, el espacio libre por donde se mueve se considera un campo potencial, los obstáculos también tendrían una carga eléctrica, pero de igual signo al robot, de manera que entre ellos se repelen y el punto de llegada tendría carga eléctrica de signo contrario al robot, es decir, se atraen [15]. De este modo, lo que provoca el movimiento del robot desde su punto de partida hasta su meta es la fuerza resultante que se genera a partir de la suma vectorial entre la fuerza repulsiva (suma total de las fuerzas de repulsión ocasionadas por los obstáculos cerca del robot) y la fuerza atractiva (asociada a la meta y al robot móvil) [16]. Lo mencionado se puede observar de manera gráfica en la Fig. 4.

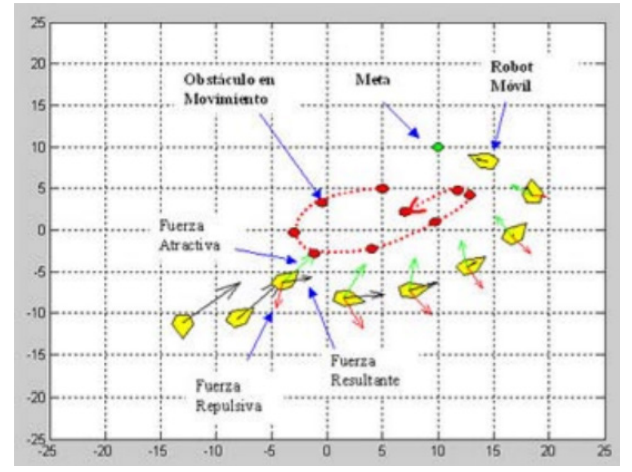

Fig. 4. Fuerzas presentes en un entorno de campos potenciales.

Dicho de otra manera, la técnica de campos potenciales para la generación de trayectorias considera el movimiento del dispositivo en un campo de fuerzas, con los obstáculos generando repulsión y la posición objetivo atracción, es decir el método sigue una técnica reactiva de navegación, ya que el movimiento del robot se encuentra guiado por el gradiente del campo potencial artificial generado [10]. Los campos pueden variar en función de la distancia del obstáculo, aunque de manera geométrica se pueden definir límites de influencia sobre estos, con el fin de evitar el cálculo de los campos de obstáculos distantes. El proceso de generación de la trayectoria consiste en hallar un camino que mantenga al dispositivo móvil lo más alejado posible de los obstáculos que se encuentren en la zona [17].

En la Fig. 5 se puede observar un ejemplo de generación de una trayectoria para un robot que pretende desplazarse desde el punto Comienzo (rojo) hasta el punto Objetivo (amarillo), teniendo como obstáculo varios polígonos dispersos en el área y los límites del espacio físico. Las ondas que se encuentran alrededor de los mismos vendrían a representar las fuerzas de repulsión que estos ejercen sobre el dispositivo [16].

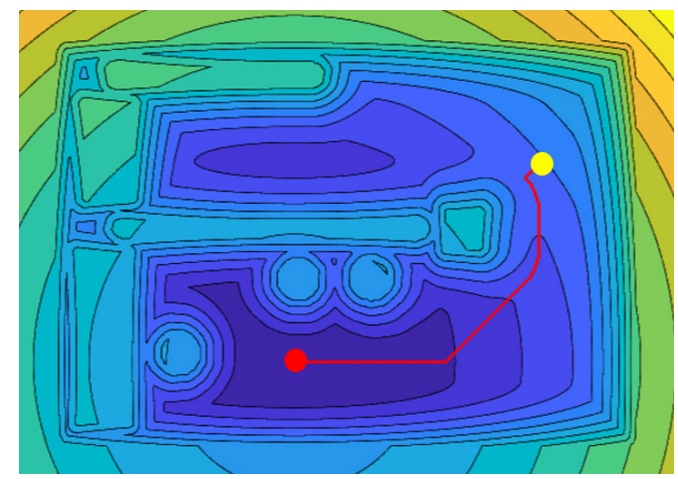

Fig. 5. Ejemplo de campos potenciales.

Para conocer más del tema en cuanto al aspecto matemático se recomienda la revisión del artículo Técnica de Navegación de Campos Potenciales para un Robot Móvil para la Evasión de Obstáculos desarrollado en el Centro de Investigación de Desarrollo Tecnológico Digital de México [15]. 


\section{C.Rapidly Exploring Random Trees}

Rapidly Exploring Random Trees es un método que permite construir un plano de trabajo en dos dimensiones donde se generan ramificaciones de exploración en un área uniforme y libre de colisión mejorando la homogeneidad de exploración y progresión hacia zonas desconocidas [18]. Se lo implementa en la robótica debido a que garantiza el descubrimiento completo y la exploración del mapa en el que se requiere que el dispositivo se desplace [4]. Independientemente de si el entorno es simple o complejo, los algoritmos RRT tradicionales siempre adaptan la longitud del paso fijo y la probabilidad de sesgo para llevar a cabo la planificación de la ruta [19] a más de que son conceptualmente simples y permiten lograr la integridad probabilística [20].

El algoritmo RRT se basa en la generación de una estructura arbórea de configuraciones que crecen a partir de un punto de origen, como se muestra en la Fig. 6. Es un algoritmo diseñado para encontrar de manera eficiente espacios no convexos de alta dimensión a través de la construcción aleatoria de ramas que rellenan un espacio determinado [21].

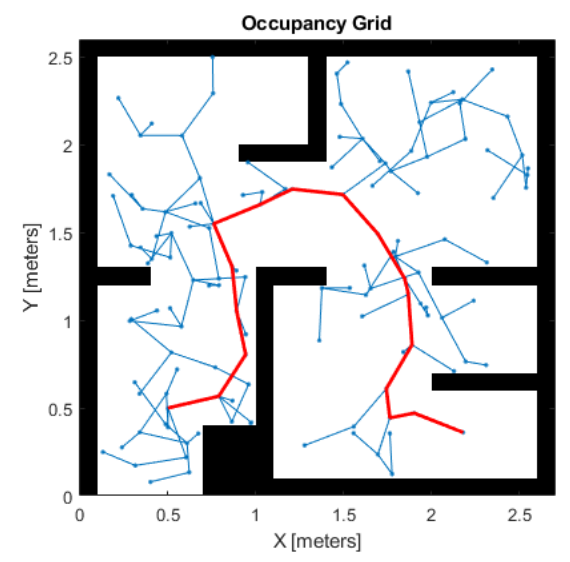

Fig. 6. Configuración del algoritmo RRT.

Estas ramas forman un árbol que se va construyendo de forma incremental a partir de muestras extraídas al azar del espacio de búsqueda, y se encuentra sesgada de manera intrínseca para crecer en grandes áreas libres de colisión. Esta particularidad convierte al RRT en un método de generación de rutas de tipo aproximado, ya que genera un muestreo aleatorio del espacio y después una subrutina de detección de colisiones que decide si las trayectorias configuradas son viables o si implican un posible choque [22].

El Rapidly Exploring Random Trees presenta una solución simple, ya que no depende de ningún parámetro inicial y favorece la exploración de toda la región para la óptima búsqueda de obstáculos. El árbol de exploración aumenta su resolución en cada iteración, y los vértices del árbol representan las configuraciones que se obtuvieron con el modelo de manera aleatoria, después de comprobar la ausencia de posibles colisiones dentro del entorno [19].

En la Fig. 7 se puede apreciar el funcionamiento del método, analizado de izquierda a derecha se tiene, en primer lugar (a) una malla de 13 x 13 que representa un espacio físico en el cual existen dos obstáculos, en segundo lugar (b) el desarrollo del árbol que define todas las posibles rutas para llegar de un punto inicial a uno final, y por último, en tercer lugar (c) se observa la selección de la ruta más corta, donde se puede observar que todas las demás que no se consideran eficientes han sido descartadas. 


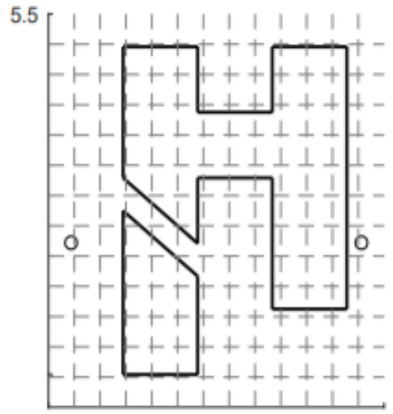

(a)

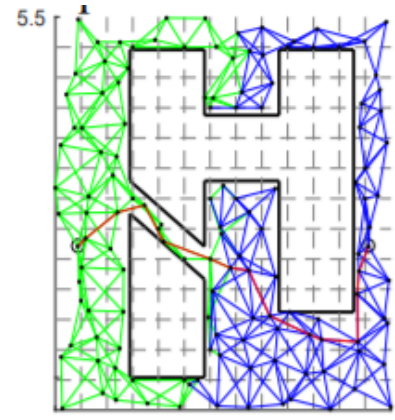

(b)

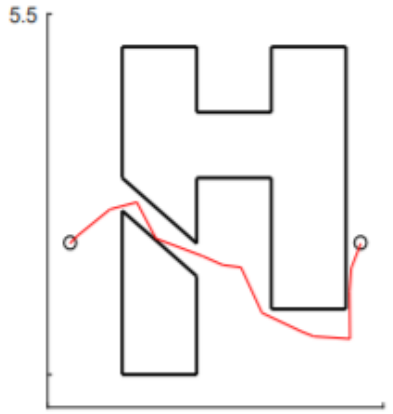

(c)

Fig. 7. Ejemplo de desarrollo del algoritmo RRT, (a) Región con obstáculos, (b) Árbol RRT, (c) Trayectoria óptima

El método RRT se desarrolla de una forma más detallada en el artículo Determinación de rutas por medio de un algoritmo RRT adaptado a una discretización del espacio de trabajo, allí se explican detalladamente las expresiones matemáticas y algorítmicas que conforman este método [21].

\section{D.Descomposición en Celdas}

El método de Descomposición en celdas es relevante en el aspecto de la robótica debido a que reduce la carga computacional de la generación de rutas mediante el proceso [23], además el deterioro del espacio de trabajo del robot es natural ya que el estado de las células tiene en cuenta una disposición abundante y adaptable. [24]. Es un método atractivo ya que es geométrico, muy consistente, fácil de entender e implementar [25].

Como su nombre lo indica, este algoritmo se basa en la descomposición de un área en celdas en las que el robot circula. La zona contiene en su interior obstáculos que el dispositivo debe rodear en caso de encontrarlos durante el avance de su trayectoria [10]. Las celdas se forman a partir de los vértices de los objetos mencionados, estas permiten al robot conocer el espacio libre, mismo que corresponde al conjunto de celdas que no contienen partes de algún obstáculo [26]. La Fig. 8 muestra un ejemplo de un espacio físico en el que se encuentran representados con polígonos los obstáculos y se tiene un punto de Inicio (Source) y Fin (Destination), para los cuales se requiere encontrar una trayectoria óptima.

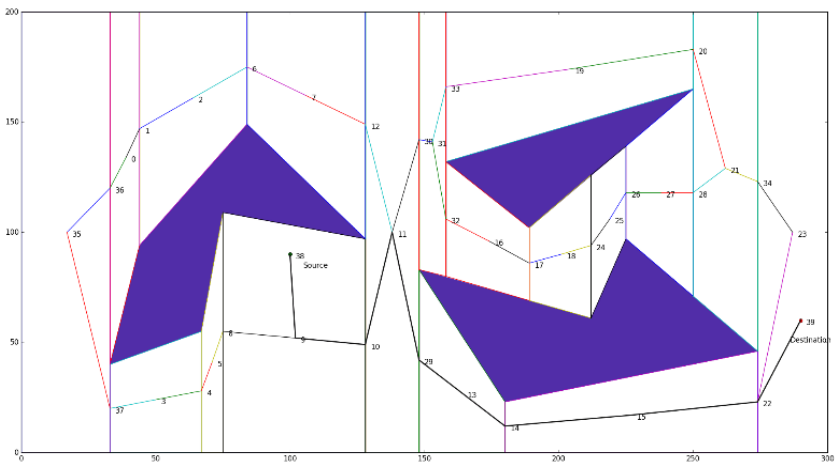

Fig. 8. Método de descomposición en celdas.

Con la descomposición en celdas, el algoritmo planificador de rutas del robot tiene la finalidad de encontrar un grafo de adyacencia en el que se indiquen cuáles de las celdas libres de obstáculos comparten una frontera en común, para determinar el espacio libre de colisión por donde el robot puede desplazarse [26]. Los nodos que se forman en el diagrama corresponden a las celdas encontradas y las aristas conectan nodos de celdas cercanas, una vez formado el grafo se busca una ruta dentro del mismo que se considere la trayectoria óptima a seguir por el dispositivo, como se muestra en la Fig. 9 resaltado con color rojo [24]. 


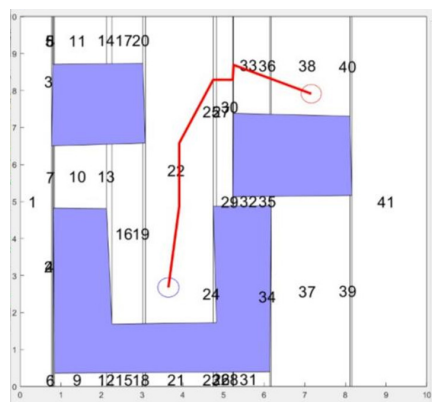

Fig 9. Trayectoria óptima encontrada con el método de descomposición en celdas.

Como se puede observar, las celdas formadas tienen una estructura simple, lo que permite que el robot se desplace entre ellas con movimiento sencillos tales como giros y avances rectilíneos, ya que una vez que el robot pasa por una celda, esta se cubre por completo, lo que reduce el método a encontrar un camino entre las celdas a través del grafo de adyacencia [25].

En caso de requerir información más detallada con respecto a este método de generación de rutas se sugiere revisar el artículo Evaluación de las técnicas de planificación de movimientos, Descomposición exacta trapezoidal, en este se podrá encontrar información específica sobre el tema y sus variantes [26].

El trabajo se estructura luego de la introducción, con el desarrollo en donde se presentan las simulaciones en el entorno estructurado y empleando las distintas metodologías. En la metodología se explican las consideraciones para el desarrollo del experimento y toma de datos. Posteriormente, en la sección resultados, se comparan las alternativas de navegación y se destacan valores relevantes para cada método. Se finaliza con las conclusiones que validan los métodos analizados.

\section{II.DESARROLLO}

Para comparar las técnicas de planificación de trayectorias, se realizaron simulaciones para cada uno de los casos "Método de planificación mediante diagramas de Voronoi", "Planificación mediante campos potenciales", "Método de planificación por descomposición de celdas", "Método Roadmap probabístico".

Para todas las simulaciones realizadas, se ha empleado un entorno de forma cuadrada y número variable de obstáculos, para lo cual un entorno poseerá 5 obstáculos de diferentes tamaños y forma similar. Otro entorno posee un número de 11 obstáculos.

A partir de simulaciones realizadas en Matlab versión estudiantil, se han obtenido las distintas trayectorias, las mismas que se presentan en la Fig. 10, donde se han representado por colores la solución de cada método empleado. Se observa una diversidad de alternativas de solución.

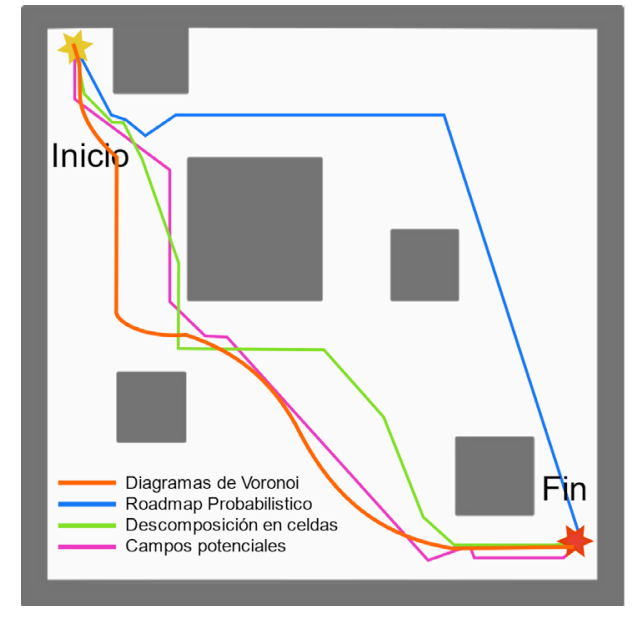

(a)

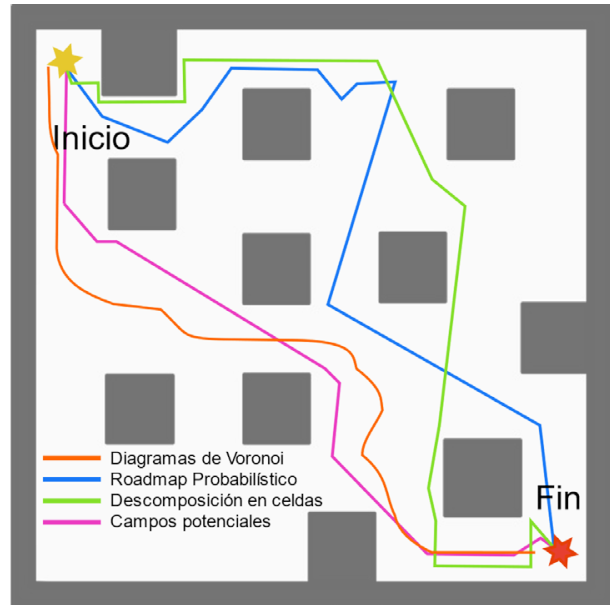

(b)

Fig.10.Solucionesdetrayectoriasgeneradaspormúltiplesmétodosdeplanificación,entornocuadradoynumero variable de obstáculos, (a) pocos obstáculos), (b) muchos obstáculos. 
Además, se ha evaluado un segundo entorno de forma rectangular para todos los métodos y con un distinto número de obstáculos, se han incorporado pequeños obstáculos a lo largo del camino a recorrer, también se ha generado irregularidades en el entorno rectangular exterior (Fig. 11) a fin de presentar a los algoritmos un entorno más variable y comparar su respuesta.

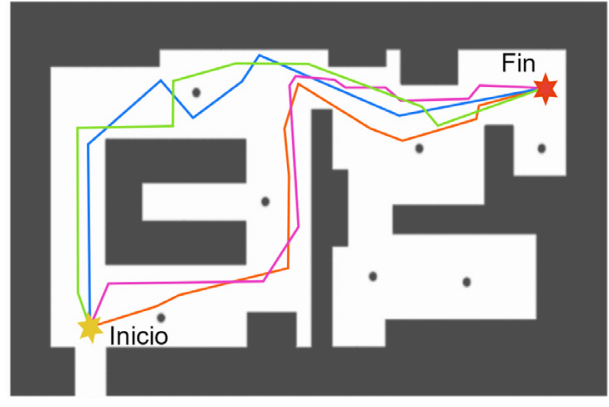

(a)

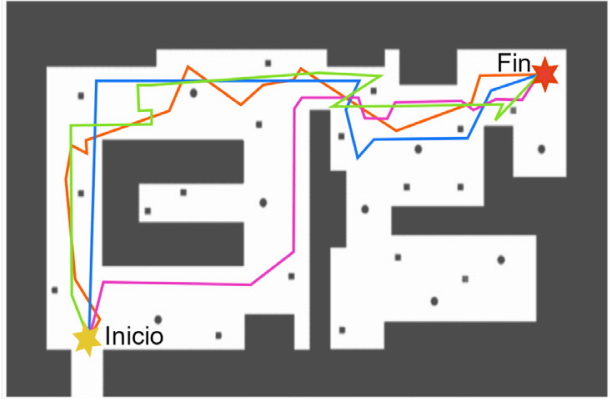

(b)

Fig.11.Soluciones detrayectoriasgeneradaspormúltiplesmétodos de planificación,entornorectangularynumero variable de obstáculos, (a) pocos obstáculos), (b) muchos obstáculos.

Para los dos entornos presentados anteriormente, el punto de Inicio y Fin coinciden para cada caso y permiten generar la trayectoria tendiendo a que esta sea extensa dentro del espacio navegable.

La TABLA I, presenta una comparación entre los tiempos de las simulaciones respectivas de cada método analizado. Se observa que, si bien, el método de Voronoi, logra las rutas más alejadas de los obstáculos, también posee un mayor tiempo de procesamiento del algoritmo.

TABLA I. Tiempos de ejecución de la tarea en múltiples métodos de planificación de trayectoria.

\begin{tabular}{|c|c|c|c|c|}
\hline \multirow[b]{2}{*}{ VORONOI } & \multicolumn{2}{|c|}{$\begin{array}{l}\text { Tiempo de procesamiento } \\
\text { Pocos obstáculos }\end{array}$} & \multicolumn{2}{|c|}{$\begin{array}{l}\text { Tiempo de procesamiento } \\
\text { Muchos obstáculos }\end{array}$} \\
\hline & 口 & $7.37 \mathrm{~s}$ & 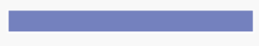 & $10.30 \mathrm{~s}$ \\
\hline RAPIDLY RAMDOM TREE & 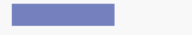 & $4.37 \mathrm{~s}$ & 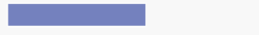 & $5.82 \mathrm{~s}$ \\
\hline CAMPOS POTENCIALES & $\square$ & $2.27 \mathrm{~s}$ & $\square$ & $2.59 \mathrm{~s}$ \\
\hline DESCOMPOSICIÓN EN CELDAS & 口 & $0.67 \mathrm{~s}$ & [ & $0.78 \mathrm{~s}$ \\
\hline
\end{tabular}

La TABLA II, además de los tiempos, presenta la complejidad del grafo considerando el número de vértices que lo componen, cuyo número varia para los distintos métodos. En adición se evalúa la optimización de la ruta considerando la longitud de la misma 
TABLAII.Comparación de tiempos de ejecución de la tarea, complejidad del grafoyoptimización de trayectoria en múltiples métodos de planificación de trayectoria.

\begin{tabular}{|c|c|c|c|c|}
\hline METODO & ENTORNO & $\begin{array}{c}\text { TIEMPO DE } \\
\text { SIMULACION (s) }\end{array}$ & $\begin{array}{l}\text { COMPLEJIDAD DEL } \\
\text { GRAFO (vértices) }\end{array}$ & $\begin{array}{l}\text { OPTIMIZACION DE } \\
\text { TRAYECTORIA (mm) }\end{array}$ \\
\hline \multirow{2}{*}{ VORONOI } & $\begin{array}{l}\text { Con pocos } \\
\text { obstáculos }\end{array}$ & 7.37 & 11 & 3331.75 \\
\hline & $\begin{array}{l}\text { Con muchos } \\
\text { obstáculos }\end{array}$ & 10.30 & 16 & 3879.17 \\
\hline \multirow{2}{*}{$\begin{array}{l}\text { RAPIDLY RANDOM } \\
\text { TREE }\end{array}$} & $\begin{array}{l}\text { Con pocos } \\
\text { obstáculos }\end{array}$ & 4.37 & 7 & 3528.82 \\
\hline & $\begin{array}{l}\text { Con muchos } \\
\text { obstáculos }\end{array}$ & 5.82 & 8 & 4036.41 \\
\hline \multirow{2}{*}{$\begin{array}{c}\text { CAMPOS } \\
\text { POTENCIALES }\end{array}$} & $\begin{array}{l}\text { Con pocos } \\
\text { obstáculos }\end{array}$ & 2.27 & 12 & 3379.70 \\
\hline & $\begin{array}{l}\text { Con muchos } \\
\text { obstáculos }\end{array}$ & 2.59 & 14 & 3473.35 \\
\hline \multirow{2}{*}{$\begin{array}{l}\text { DESCOMPOSICIÓN } \\
\text { DE CELDAS }\end{array}$} & $\begin{array}{l}\text { Con pocos } \\
\text { obstáculos }\end{array}$ & 0.67 & 9 & 3642.83 \\
\hline & $\begin{array}{l}\text { Con muchos } \\
\text { obstáculos }\end{array}$ & 0.78 & 12 & 4425.66 \\
\hline
\end{tabular}

\section{III.METODOLOGÍA}

Para la obtención de los gráficos que representan las simulaciones del apartado anterior, se utilizaron algunos simuladores que han partido de algunos desarrollos de carácter académicos como [12], [27] para la generación de la trayectoria con el uso de diagramas de Voronoi, el método de [13] para su uso mediante campos potenciales, [26] para la determinación mediante descomposición en celdas y Roadmap probabilístico.

Se ha considerado para representar el desempeño de los métodos de "Roadmap Probabilisticos", el uso del algoritmo Rapidly Ramdom Tree, mismo que se encuentra en potencial desarrollo y uso para navegación tanto en dos como en tres dimensiones.

En las simulaciones realizadas, se adaptaron los entornos a fin de que coincidan en dimensión y complejidad debido al número y disposición de los obstáculos. Se obtuvo la información del procesamiento y con ello el tiempo de cómputo de los algoritmos analizados.

\section{IV.RESULTADOS}

Respecto del tiempo de simulación, se observa que el método de Voronoi ocupa un tiempo mayor en el procesamiento, esto debido a que su trayectoria toma en cuenta el cálculo de las distancias intermedias y más alejadas de los obstáculos, se evidencia que a pesar de generar una ruta muy segura y lejos de producir colisiones, este puede tener sus inconvenientes de retardo en robot de baja capacidad de procesamiento.

Por otra parte, el grafo generado por el método de descomposición en celdas, logra obtener la trayectoria en un tiempo muy reducido respecto de los otros métodos. Esto se debe a que el número de puntos con los que trabaja este método, es muy inferior al de Voronoi.

Los tiempos presentados corresponden a la media de los valores ensayados en ambos entornos en corridas sucesivas. Sn embargo el método Rapidly Ramdom Tree, en su carácter de aleatorio, genera algunas alternativas, las cuales, a su vez, se promediaron. Los demás métodos poseían soluciones únicas para las configuraciones propuestas. Se evidencia que la respuesta de todos los métodos es independiente en gran manera del número de obstáculos en el entorno, siempre que estos se encuentren a distancias considerablemente mayores relativas al tamaño de los obstáculos presentados.

En cuanto a la complejidad de los grafos, los gráficos más complejos se presentaron empleando los métodos de Voronoi y Descomposición en celdas. Esto puede explicarse debido a que el grafo es función directa del número de vértices del entorno. Para el caso del método Rapidly Random Tree, el número de vértices es función de la característica aleatoria con la que se generan las posibilidades de navegación en el espacio libre. Por otra parte, el método de Campos potenciales, considera a los obstáculos como zonas que permiten un cálculo de la trayectoria 
adecuada para alcanzar el objetivo independientemente del número de vértices de los obstáculos.

Para la optimización de la trayectoria, se ha considerado la longitud de la misma para todos los casos, estos valores se presentan en la TABLA I. Se ha considerado la longitud como un indicativo de esta optimización dejando de lado la energía empleada para la navegación debido al cambio brusco en la trayectoria, cuyo análisis en este documento no es tomado en cuenta.

\section{V.CONCLUSIONES}

Obtener rutas más alejadas de los obstáculos es una adecuada alternativa de navegación, como en el caso del método de diagramas de Voronoi, aunque también exige una mayor velocidad de procesamiento, esto es un factor importante para implementar este algoritmo en robots móviles.

El método de descomposición por celdas, por otra parte, exige menos recursos computacionales cuando el entorno se presenta con pocos obstáculos, mientras que, con un mayor número de ellos, el método de campos potenciales posee un mejor desempeño en este ámbito con la posibilidad de ser adatado además para entornos dinámicos, cuya ventaja es única frente a los otros métodos. Respecto de la reacción de los métodos frente al entorno, es evidente que obstáculos en medio del espacio libre, independientemente del tamaño de los mismos, modifican mucho más la trayectoria, que irregularidades en las proximidades del entorno circundante.

La complejidad de los gráficos en los métodos de Voronoi y Campos potenciales, generan trayectorias que si bien, reducen la probabilidad de colisión con los obstáculos, también representan un mayor esfuerzo de control para lograr conservar la trayectoria variable presentada.

\section{REFERENCIAS}

[1]H. Ajeil., K. Ibraheem, A. Sahib., J. Humaidi., "Multi-objective path planning of an autonomous mobile robot using hybrid PSO-MFB optimization algorithm, ” Applied Soft Computing, vol. 89, April 2020.

[2]K.Patle., G. Babu., A. Pandey., D.R.K. Parhi., A. Jagadeesh., "A review: On path planning strategies for navigation of mobile robot," Defence Technology, vol. 15, pp. 582-606, August 2019.

[3]T. Mack., C. Copot., D. Trung., R. De Keyser., "Heuristic approaches in robot path planning: A survey," Robotics and Autonomous Systems, vol. 86, pp. 13-28, December 2016.

[4]L. Zhang., Z. Lin., J. Wang., B. He., "Rapidly-exploring Random Trees multi-robot map exploration under optimization framework," Robotics and Autonomous Systems, vol. 131, 2020.

[5]S. Khan and M. K. Ahmmed, "Where am I? Autonomous navigation system of a mobile robot in an unknown environment," 2016 5th International Conference on Informatics, Electronics and Vision (ICIEV), pp. 56-61, December 2016.

[6]V. Castro, J. P. Neira, C. L. Rueda, J. C. Villamizar and L. Angel, "Autonomous Navigation Strategies for Mobile Robots using a Probabilistic Neural Network (PNN)," IECON 2007 - 33rd Annual Conference of the IEEE Industrial Electronics Society, pp. 2795-2800, Taipei, 2007.

[7]Y. Li., W. Wei., Y. Gao., D. Wang., C. Fan., "PQ-RRT*: An improved path planning algorithm for mobile robots," Expert Systems with Applications, vol. 152, August 2020.

[8]A. Muñoz., "Generación global de trayectorias para robots móviles, basada en curvas betaspline," Dep. Ingeniería de Sistemas y Automática Escuela Técnica Superior de Ingeniería Universidad de Sevilla, 2014.

[9]H. Montiel, E. Jacinto, H. Martínez, “Generación de Ruta Óptima para Robots Móviles a Partir de Segmentación de Imágenes,” Información Tecnológica, vol. 26, 2015.

[10] C. Expósito, "Los diagramas de Vornooi, la forma matemática de dividir el mundo," Dialnet, Diciembre 2016.

[11]N. Sotomayor., A. Yandún., "Planeación y seguimiento de trayectorias para un robot móvil," Researchgate, Agosto 2012.

[12]Y. Zhang, Z. Liu and L. Chang, "A new adaptive artificial potential field and rolling window method for mobile robot path planning," 2017 29th Chinese Control And Decision Conference (CCDC), pp. 7144-7148 July, 2017. [13]S. Garrido., L. Moreno., D. Blanco, P. Jurewicz., "Path Planning for Mobile Robot Navigation using Voronoi Diagram and Fast Marching," International Journal of Robotics and Automation (IJRA), vol. 2, 2011.

[14]Q. Jia, X. Wang, "An improved potential field method for path planning," 2010 Chinese Control and Decision Conference, pp. 2265-2270, 2010.

[15]T. Osuna, L. Gonzáles y L. Aguilar, “Técnica de navegación de campos potenciales para un robot móvil para 
la evasión de obstáculos", Marzo, 2010.

[16]V. Gonzáles y R. Parkin, "Evadiendo obstáculos con robots móviles”, Enero 2005.

[17]J. Sfeir, M. Saad and H. Saliah-Hassane, "An improved Artificial Potential Field approach to real-time mobile robot path planning in an unknown environment," 2011 IEEE International Symposium on Robotic and Sensors Environments (ROSE), pp. 208-213, 2011.

[18]I. Pérez-Hurtado, M. J. Pérez-Jiménez, G. Zhang and D. Orellana-Martín, "Robot path planning using rapidly-exploring random trees: A membrane computing approach," 2018 7th International Conference on Computers Communications and Control (ICCCC), pp. 37-46, 2018.

[19]S. Zhang, J. Pu, Y. Si., L. Sun, "Smooth Path Planning for Mobile Robot Based on Adaptive Rapidly-exploring Random Tree*," 2018 IEEE International Conference on Information and Automation (ICIA), pp. 591-596, 2018. [20]Y. Ying, Z. Li, G. Ruihong, H. Yisa, T. Haiyan and M. Junxi, "Path planning of mobile robot based on Improved RRT Algorithm," 2019 Chinese Automation Congress (CAC), Hangzhou, pp. 4741-4746, 2019.

[21]C. Calderón, T. Bustillo, "Determinación de rutas por medio de un algoritmo RRT adaptado a un discretización del espacio de trabajo", Junio 2018.

[22]D. López, F. Gómez, F. Cuesta y A. Ollero, "Planificación de trayectorias con el algoritmo RRT. Aplicación a robots no holónomos", Enero, 2010.

[23]JW Kang, SJ Kim, MJ Chung, H. Myung, JH Park y SW Bang, "Planificación de ruta para la operación de cobertura completa y eficiente de robots móviles", Conferencia Internacional de 2007 sobre Mecatrónica y Automatización, pp. 2126-2131, 2007.

[24]N. Zafara, J.C.Mohanta, "Methodology for Path Planning and Optimization of Mobile Robots: A Review," Procedia Computer Science, vol. 133,pp. 141-152, 2018.

[25]H. A. Vasseur, F. G. Pin and J. R. Taylor, "Navigation of a car-like mobile robot using a decomposition of the environment in convex cells," Proceedings. 1991 IEEE International Conference on Robotics and Automation, vol. 2, pp. 1496-1502, 1991.

[26]C. Vélez, J. Guzmán y I. Durley, "Evaluación de las técnicas de planificación de movimientos, descomposición exacta trapezoidal y descomposición adaptativa de celdas a través de mallas”. Dialnet, Marzo, 2012.

[27]W. Regli, "Robot Lab: Robot path planning," Lecture notes of department of computer science, Drexel University, Oct 2007.

\section{RESUMEN CURRICULAR}

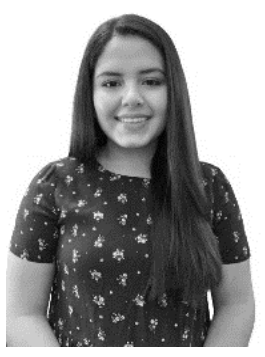

Gabriela Alvarez, Estudiante de Ingeniería en Producción Industrial de octavo semestre de la Universidad de las Américas en Quito - Ecuador. Considerada una alumna destacada, por lo que es ayudante de cátedra de varias materias de la carrera y de su coordinación. Con participación en diversas conferencias sobre la industria nacional e internacional.

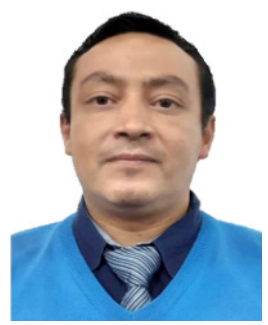

Omar Flor, Docente-Investigador de la Universidad de las Américas, especialista en diseño mecánico, especialista en diseño de equipos médicos. 